\title{
Le contrôle de la $\beta$-oxydation peroxysomiale par des membres de la superfamille des récepteurs nucléaires
}

Les peroxysomes sont des organites intracellulaires intervenant dans plusieurs fonctions métaboliques importantes, notamment la $\beta$-oxydation. Toute une série de composés organiques provoquent une prolifération des peroxysomes, et par conséquent une augmentation de la $\beta$-oxydation des lipides à très longue chaîne qui explique, en partie, l'effet hypolipémiant de médicaments appartenant à la famille des fibrates [1]. En 1990, l'équipe de S. Green (Macclesficld, GB) rapportait le clonage chez la souris d'un ADNc codant pour une molécule appelée PPAR (peroxisome proliferator activated receptor) qui pouvait être activée par des inducteurs de la prolifération peroxysomiale $\left(\mathrm{m} / \mathrm{s} n^{\circ} 10\right.$, vol. 6, p. 1 017). Quoique cette molécule PPAR appartînt à la famille des récepteurs nucléaires, la séquence cible d'ADN à laquelle elle se lie ainsi que le ligand exact capable de l'activer restaient indéterminés. En effet, Issemann et Green avaient utilisé, pour démontrer les effets de PPAR, un récepteur hybride comportant le domaine de liaison présomptif du ligand de PPAR et le domaine de liaison à l'ADN d'un récepteur stéroïdien. Lorsqu'un vecteur d'expression commandant la synthèse de cette protéine hybride était introduit par transfection dans des cellules en même temps qu'un gène test contrôlé par des éléments de réponse aux stéroïdes, ce dernier était activé lors du traitement des cellules par des inducteurs de la prolifération peroxysomiale. Ces derniers agissaient, cependant, indirectement car ils étaient tout à fait incapables de se lier directement aux récepteurs PPAR. Le groupe de S. Green vient maintenant de démontrer que le récepteur PPAR murin peut directement activer l'un des gènes clé de la $\beta$-oxydation peroxysomiale, celui de l'acyl-CoA oxydase, en se fixant directement à un élément de réponse PPRE (peroxisome proliferator response element) situé $570 \mathrm{pb}$ en amont du début du gène [2]. Cet élément PPRE semble composé de plusieurs répétitions, directes et inversées, de l'hexanucléotide TGACCT et TGTCCT. En fait, c'est la répétition directe de deux de ces éléments séparés par une seule base qui semble conférer la réponse aux inducteurs de prolifération peroxysomiale. Ces séquences cibles sont proches des autres éléments de réponse fixant des récepteurs de cette superfamille $\left(\mathrm{m} / \mathrm{s} n^{\circ} 3\right.$, vol. 6, p. 307 et $n^{\circ} 8$, vol. 7, p. 863). Plus particulièrement, on trouve dans l'élément de réponse aux rétinoïdes du gène de la CRBP2 (cellular retinol binding protein 2), démontré être un site de liaison pour des molécules RXR, une semblable répétition directe $d$ 'hexamères identiques, mais en position inversée $(\mathrm{m} / \mathrm{s}$ $n^{\circ} 8$, vol. 7, p. 863). Des études complémentaires sont manifestement indispensables pour que soient précisées les caractéristiques structurales des éléments de réponse conférant, soit la sensibilité aux rétinoïdes, soit celle aux inducteurs de prolifération peroxysomiale. Indépendamment, le laboratoire de W. Wahli (Lausanne, Suisse) en collaboration avec celui de C. Dreyer (Tübingen, Allemagne), vient d'isoler trois clones de type PPAR à partir des banques d'ADN complémentaire de xénope. Ces molécules, appelées xPPAR $\alpha \beta$ et $\gamma$, sont plus ou moins spécifiques de tissu et de stades du développement [3]. Elles ont la même capacité que le PPAR murin à être activé par les fibrates et stimulent également la transcription du gène de l'acyl-CoA oxydase. L'élément de réponse est bien celui décrit par $\mathrm{S}$. Green au niveau du gène murin. Les trois molécules xPPAR identifiées par C. Dreyer et al. semblent reconnaî- tre le même élément de réponse PPRE avec une différence importante entre xPPAR $\alpha$ et les deux autres molécules : alors que le PPAR murin de S. Green aussi bien que les PPAR $\beta$ et $\gamma$ de xénope de $W$. Wahli sont stimulés par les inducteurs de prolifération peroxysomiale, l'activation transcriptionnelle conférée par xPPAR $\alpha$ est indépendante de ces inducteurs. Il est possible que ce dernier type de récepteurs reconnaisse, en fait, un ligand endogène, ou bien soit constitutivement activé. La spécificité d'expression selon le stade du développement et les tissus notés pour les PPAR de xénope suggère que ces différentes molécules pourraient jouer des rôles très particuliers dans différents contextes cellulaires, reconnaissant peut-être différentes séquences cibles d'ADN et étant activécs par des ligands variés. Un tel schéma est maintenant bien classique pour ce qui est des récepteurs des rétinoïdes RAR et RXR (m/s $n^{\circ} 3$, vol. 8 , p. 281). Des travaux ultérieurs diront si des récepteurs PPAR, comme les récepteurs de l'acide rétinoïque, de la vitamine $\mathrm{D}$ et des hormones thyroïdiennes, à la famille desquels ils appartiennent, agissent sous la forme d'hétérodimères, peut-être avec des molécules RXR. Restera également à déterminer quels sont les véritables ligands, si ligands il y a, des PPAR : produits de métabolismes, des inducteurs de prolifération peroxysomiale, peut-être dépendants du cytochrome P450? Ligands endogènes dont la synthèse serait stimulée par ces derniers? Ou encore, en l'absence de vrais ligands, processus d'activation post-traductionnelle induite par les inducteurs? 
1. Latruffe N. Les peroxysomes et la prolifération cellulaire ou la prise en considération d'un organite méconnu. médecine/sciences $1992 ; 8$ : 238-9.

2. Tugwood JD, Issemann I, Anderson RG, Bundell KR, Mc Pheat WL, Green S. The mouse peroxysome proliferator activated receptor recognizes a response element in the 5' flanking sequence of the rat acyl-CoA oxidase gene. EMBO J $1992 ; 11: 433-7$.

3. Dreyer C, Krey G, Keller H, Givel F, Helftenbein G, Wahli W. Control of the peroxisomal $\beta$-oxidation pathway by a novel family of nuclear hormone receptors. Cell 1992; 68: 879-87.

\section{La revue Raison Présente} et le cercle

Recherche et Critique

14, rue de l'École-Polytechnique vous invitent

à leur cycle de conférences 1992

\section{La Maîtrise du Vivant}

\section{Lundi 16 mars :}

Où en sont les techniques?

E.E. Baulieu (Académie des Sciences, université Paris-Sud, INSERM, hôpital de Bicêtre). A. Kahn (INSERM, hôpital Cochin). E. Papiernik (université René-Descartes, hôpital Baudelocque).

\section{Lundi 23 mars :}

Peut-on modifier l'homme? H. Caillavet (ancien ministre). E. Papiemik (université René-Descartes, hôpital Baudelocque). P.A. Taguieff (CNRS, sociologie).

\section{Lundi 30 mars :}

Réflexions sur les fondements de la bioéthique

A. Memmi (université de Nanterre, écrivain). D. Jacquemin (faculté de médecine de Louvain). J.P. Thomas (philosophe).

\section{Lundi 6 avril :}

Comités d'éthique et démocratie G. Braibant (Conseil d'État). H. Atlan (université $P$. et M.-Curie, hôpital de l'Hôtel-Dieu). A. Langaney (Muséum National d'Histoire Naturelle).

Pour chaque conférence, la première personnalité citée introduira et dingera le débat. Les conférences commenceront à 18 heures précises au Collège de France, salle 8, Place Marcelin-Berthelot (rue des Écoles), 75005 Paris.
75005 Paris. Tél. : 46.33.03.50

\section{COURRIER}

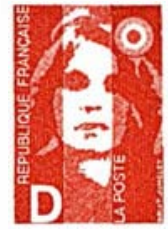

Dans son éditorial [1] $\left(m / s n^{\circ} 1\right.$, vol. 8 , p. 8) consacré à "l'épidémiologie du

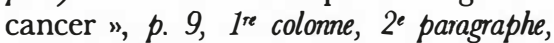
Maurice Tubiana formule ce qu'il conviendrait d'appeler des propos de circonstance, c'est-à-dire ne rapportant pas la réalité objective. En mettant sur le compte de l'allongement de l'espérance de vie, l'accroissement de l'incidence des cancers et de la mortalité par cancer, il évite soigneusement d'énoncer une vérité simple, même si elle est désolante, à savoir : en distinguant sur des populations normalisées pour l'âge la fréquence par classe d'âge de la fréquence absolue, la mortalité par cancer a régressé pour les classes de moins de trente ans, a progressé pour les classes de plus de trente ans entre 1950 et 1985 , de manière à pcine différente aux États-Unis, en Allemagne, et en France, pays pour lesquels on dispose de travaux publiés [2-5] qui sont tous aussi clairement explicites.

Bien entendu, l'accroissement des cancers du poumon et du sein, la régression des cancers du col utérin et de l'estomac s'observent dans les trois pays pendant la période considérée. Pour les cancers fréquents de l'adulte, aucun progrès thérapeutique ou préventif n'a pu influencer favorablement la situation.

Maurice Tubiana n'est pas seul à s'exprimer ainsi, il serait néanmoins fâcheux que médecine/sciences accréditc cette inexactitude sur laquelle sont fondées des campagnes publiques et l'attribution des crédits. Conviendraitil de manipuler l'opinion plutôt que de reformuler les hypothèses de travail sur lesquelles les chercheurs font porter leur effort?

Jean-Claude Salomon

Directeur de Recherches au Cnrs, BP8, 7, nue Guy Moquet, 94801 Villejuif Cedex, France.
1. Tubiana M. L'épidémiologie du cancer. méde-

2. Special Report. Measurement of progress against cancer. J Natl Cancer Inst 1990; 82 : 825-35.

3. Bailar JC III, Smith EM. Special articles: progress against cancer? N Engl J Med 1986 314: 1226-32.

4. Hill C, Benhamou E, Doyon F, Flamant R. Évolution de la mortalité par cancer en France entre 1950 et 1985. Paris : Éditions INSERM, 1989.

5. Becker N, Smith EM, Wahrendorf J. Time trends in cancer mortality in the Federal Republic of Germany : progress against cancer? Int $J$ Cancer 1989 ; 43 : 245-9. cine/sciences $1992 ; 8: 8-9$.

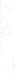

$m / s n^{\circ} 3$, wol. 8 , mars 92 